\title{
Mathematical modelling, nonlinear dynamics, bifurcation, synchronization and control of mechanisms driven by power supply
}

\author{
José Manoel Balthazar ${ }^{1,3, a}$, Paulo Batista Gonçalves ${ }^{2}$, Angelo Marcelo Tusset ${ }^{3}$, Grzegorz Litak $^{4}$, \\ Átila Madureira Bueno ${ }^{5}$, and Mauricio Aparecido Ribeiro ${ }^{3}$ \\ 1 School of Engineering, São Paulo State University - UNESP, Bauru, SP, Brazil \\ 2 Pontifical Catholic University, Rio de Janeiro, RJ, Brazil \\ 3 Federal University of Technology - UTFPR, Ponta Grossa, PR, Brazil \\ 4 Lublin University of Technology, Lublin, Poland \\ ${ }^{5}$ Institute of Science and Technology, São Paulo State University - UNESP, Sorocaba, SP, Brazil
}

Published online 21 October 2021

(C) The Author(s), under exclusive licence to EDP Sciences, Springer-Verlag GmbH Germany, part of Springer Nature 2021

\begin{abstract}
The special issue entitled "Mathematical modelling, nonlinear dynamics, bifurcation, synchronization and control of mechanisms driven by power supply," published by The European Physical Journal: Special Topics (EPJST), aims to bring into discussion works involving nonlinear and dynamic and chaotic behaviors, which are frequently found in mechanical and electromechanical systems, nonlinear circuits, and biologically inspired systems, stimulating the integration of findings on the nonlinear dynamics that can be observed in various systems in the physical sciences and engineering. This special issue presents 27 papers concerning the dynamics and control of flexible structure systems, nonideal systems, energy harvesting problems, and vibration absorbers. In addition, the papers address problems involving chaos control of nonlinear oscillations, phase-locked loops, synchronization of nonlinear oscillators, dynamics of bio-inspired and biostructural systems, nonlinear methods, and nonlinear circuits.
\end{abstract}

\section{Preliminary comments}

The main difficulty in investigating nonlinear systems in comparison with linear systems is the absence of the superposition principle, which allows us to discretize any linear problem using a small number of essential modes and decouple the linear equations of motion. To overcome this drawback, several methods for obtaining significantly reduced-order modes for nonlinear systems have been proposed in recent decades, including normal forms, nonlinear normal modes, and proper orthogonal decomposition, in addition to the classical perturbation methods. Every nonlinear vibrating system must be solved individually, and a specific methodology must be developed for each class of problems. Many oscillatory (vibrating) problems involving real systems are inherently nonlinear and cannot be explained by or solved on the basis of linear vibration theory. As such, the mathematical modelling of nonlinear dynamical systems and tools for their analysis are becoming increasingly important in the engineering sciences and applied mechanics. Among nonlinear dynamical systems, those driven by a power supply are found in diverse applications.

Nonlinear dynamics has its origins in the famous "three-body problem." It is well known that a non-

\footnotetext{
${ }^{\mathrm{a}}$ e-mail: jmbaltha@gmail.com (corresponding author)
}

linear system is any system in which the change in the output is not proportional to the change in the input. These systems display a wealth of phenomena not found in linear systems, such as coexisting solutions, sub- and super-harmonic oscillations, hardening and softening behavior, synchronization, bifurcations including dynamic jumps, period-doubling, crises, coexisting bifurcation branches, hysteresis, and chaos, where small changes in initial conditions can lead to vastly different outcomes. These phenomena are fully explored in the present special issue.

Among the important analytical tools used in nonlinear dynamics are phase portraits, Poincaré maps, frequency spectra, basins of attraction, Lyapunov exponents, nonlinear time series, and bifurcation diagrams. Bifurcation diagrams can comprehensively explain the changes in the nonlinear dynamic response and stability of solutions over a range of parameter values, making them an essential tool for understanding nonlinear problems.

We wish to thank all the authors for their enthusiastic response and hard work, and for supporting our idea. The special issue was open to review papers and technical papers alike. We hope that this volume can contribute to constructive discussions on the mathematical modelling, nonlinear dynamics, bifurcation, syn- 
chronization, and control of systems driven by power supply and stimulate further research in the field. We sought high-quality original research focusing on novel advances regarding the theme of this special issue, containing 27 papers selected from 49 submitted contributions.

\section{Contributed papers in this special issue}

The 27 selected contributions in this special issue shed light on a range of interesting problems related to the mathematical modelling, nonlinear dynamics, bifurcation, synchronization, and control of systems driven by power supply, including a number of interesting applications in several engineering fields. Fellah et al. [1] propose a new chaotic generator circuit intended to achieve an ultra-wideband range occupied by a chaotic signal with low-cost design. The architecture of the proposed circuit is simulated using the advanced design systems (ADS) tool, and a mathematical model of the circuit is developed. Based on simulation results, the proposed circuit is manufactured using a positive photosensitive PTFE copper board (ABT18), and experimental results show that the circuit demonstrates good chaotic dynamic behavior, with an ultra-wideband signal up to $5 \mathrm{GHz}$ and uniform spectrum of around $40 \mathrm{dBm}$. The random-like signal generated is especially useful in many real-world applications such as jamming and through-the-wall imaging.

Piqueira [2] demonstrates that Melnikov's method, originally proposed to identify chaos in dynamic systems, can be successfully used to establish appropriate circuit parameters by determining the regions that ensure proper operation under conditions of noise and nonlinearity, represented by periodic perturbations. The study considers the use of one of the most frequently adopted configurations for clock recovery - the second-order PLL with lag-lead filter - and provides a valuable tool for circuit dimensioning and the design of clock distribution networks.

Daum et al. [3] investigate the dynamical behavior of a four-dimensional magnetohydrodynamic model, consisting of a generalized Lorenz model using Lyapunov exponents and bifurcation diagrams, and focusing on the chaotic and hyperchaotic behaviors associated with the bifurcation parameter that couples the equations of fluid displacement to the induced magnetic field. The state-dependent Riccati equation (SDRE) and optimal linear feedback control (OLFC) techniques are considered to design the state feedback control system to stabilize the system in a previously defined orbit. The performance of the two control approaches is then compared, showing that the OLFC delivers better results.

Azzaz et al. [4] present a new way of designing multiwing chaotic systems. The proposed design is based on a 3D continuous chaotic Lorenz system, improved by introducing saw-tooth and sine functions. The basic properties of the proposed system are analyzed using equilibrium points, a phase portrait, Lyapunov expo- nent, and bifurcation diagram. The proposed system is then used to design a new chaos-based true random number generator (TRNG) by analyzing its chaotic dynamical behavior and a field-programmable gate array (FPGA) implementation. The proposed hardware architecture is based on two stages of pipeline and a parallel structure (only two clock cycles). Experimental implementation results show that the design can achieve a maximum operating frequency of 12:649 MHz and a throughput of $202 \mathrm{Mbit} / \mathrm{s}$. Moreover, the random bit sequences produced by the TRNG have successfully passed the NIST-800-22 statistical standards tests. The proposed multi-wing attractor also manifests complex dynamics and can be employed in many engineering applications, especially in embedded cryptographic contexts.

Sun et al. [5] propose a novel 3D memristor-based time-delay (MBTD) chaotic system with extreme multistability and line equilibrium points, which they apply in a pseudo-random sequence generator. Firstly, nonlinear phenomena are observed using two-parameter bifurcation diagrams, such as coexisting hidden attractors of both periodic and chaotic nature, coexisting multi-scroll hidden attractors, coexisting singlescroll hidden attractors, and coexisting periodic attractors, resulting in hidden extreme multi-stability. Moreover, the system, which displays good autocorrelation and cross-correlation, is applied to generate a chaotic pseudo-random sequence. It is shown that the approximate entropy of the chaotic pseudo-random sequence is greater than that of other time-delay chaotic systems.

The work presented by Piccirillo [6] investigates the control of the basin erosion phenomenon in a vibrating system with limited power supply (nonideal excitation). To achieve this goal, a linear vibration absorber (LVA) is employed. The effect of this passive control method is to shift the erosion profiles towards larger values of the applied voltage in the armature of the DC motor, which is a control parameter of the nonideal system. Numerical results show that this procedure can offer a good alternative to increase the dynamic integrity in mechanical systems with more than one degree of freedom.

Oliveira and Gonçalves [7] propose a 2D spatial model of human walking during the single stance phase (SSP), using a flat foot adaptation. The model is based on inverse dynamics in which the angular displacements are interpolated by fifth-degree B-splines. The equation of motion is developed using the recursive Lagrangian formulation due to its computational efficiency. An optimization problem is set up to obtain the control points of the B-splines using the dynamic effort, which is a limited power supply, as the objective function. The constraints imposed on the motion are both time-dependent (such as the torque/angle limits and the dynamic stability criterion defined by the zero moment point $[\mathrm{ZMP}]$ ) and time-independent (such as the equation of motion and step length). All the steps are explained in detail to find the dynamics of the body using the smallest number of laboratory results. The solution from the model is found to compare favor- 
ably to Winter's data, in particular the ground reaction forces, which are important in many engineering applications.

In Lacerda et al. [8], the authors derive a model to study and simulate the dynamics of power grids that, after a few approximations, turns out to be the secondorder Kuramoto model. They then use this model to perform several numerical simulations related to the stability and vulnerability of energy transmission networks. With regard to the stability, assuming the grid is fully functional and, therefore, is in a synchronous state, they analyze how the grid responds to large perturbations and how this response can be influenced by the location of the node being perturbed. They also show how a simple change in the topology or transmission capacity of a line can affect the synchronization of the grid. With regard to the vulnerability, several indexes for identifying dynamically vulnerable nodes and edges are presented.

Rizvi et al. [9] study multi-waves, homoclinic breathers, and M-shaped rational and periodic cross-kink solutions for the coupled Higgs equation (CHE) by using distinct transformations. They obtain these solutions with the aid of logarithmic transformations and symbolic computations. Moreover, they derive a number of soliton wave solutions for the CHE in polynomial form. These solutions include solitary waves, soliton waves, and Jacobi elliptic function solutions.

Guo and Luo [10] assess the periodic responses of a nonlinear oscillator with an electromagnetic resonant shunt tuned mass damper inerter (ERS-TMDI). The symmetric and asymmetric periodic motions of such a system are obtained through an implicit mapping method. The stability and bifurcation analysis of periodic motions are complemented by an eigenvalue analysis. The frequency-amplitude characteristics of periodic motions are presented using the finite Fourier series analysis to gain a better understanding of nonlinear responses of the nonlinear oscillator. Numerical simulations of the stable periodic motions are subsequently presented to verify the semi-analytical predictions of symmetric and asymmetric periodic motions. As is shown, the asymmetric periodic motions cannot be readily obtained using traditional methods. The frequency-amplitude characteristics of symmetric and asymmetric periodic motions are particularly well suited to the design of electromagnetic resonant shunt tuned mass damper inerters. The results presented here can also be applied to wind-induced vibration, earthquake building vibration reduction, and energy harvesting.

Telem et al. [11] consider the dynamics of a system of two coupled van der Pol oscillators whose (inversion) symmetry is broken by a constant excitation force. They investigate the bifurcation structures of the system both with respect to its parameters and the intensity of the excitation force, and by using numerical methods. They find that the forced system experiences rich and complex bifurcation patterns including perioddoubling, crises, coexisting bifurcation branches, and hysteresis. Due to the absence of symmetry, the sys- tem develops relatively complex dynamics, which are reflected in the coexistence of multiple (i.e., two, three, or four) asymmetric attractors. Moreover, one of the most interesting and striking features of the system considered here is the coexistence of periodic and chaotic bubbles of bifurcation for certain parameter ranges. This latter phenomenon has never been reported before.

Semenov et al. [12] investigate self-oscillations in a system with hysteresis described by a modified van der Pol equation which is formalized within the Preisach approach. The system considered here is a mathematical model of an electrical system similar to the classical van der Pol system, where characteristics of the nonlinear part are of a hysteretic nature. The main method for studying this system is the classical small parameter approach. Using this method, an analytical solution of the equation describing the system under consideration is obtained. Numerical results are presented, and an analysis comparing the dynamics of the system with those of the classical van der Pol oscillator is carried out. The dynamics modes of the modified oscillator are investigated depending on the parameters of the system. Lastly, the spectral characteristics are also compared with the corresponding characteristics of the classical van der Pol oscillator.

Hedrih [13] analyzes the free and forced oscillatory motions of a model of a hybrid biostructure and investigates their stability with respect to the geometrical and kinematic parameters of the model. The basic biostructure is modelled as a cantilever of circular cross-section with rigidly connected structures and discrete masses, and has three degrees of freedom. To describe the oscillatory behavior of this hybrid biostructure, a system of coupled differential equations and displacement influence coefficients of the cantilever are used. For specific geometrical parameters of the hybrid biostructure, one unstable mode of free oscillations is identified. Amplitude-frequency graphs for forced oscillations for specific geometrical parameters, and for both stable and unstable modes, are presented. Conditions for resonance and dynamical absorption are also discussed.

Borowiec et al. [14] propose a new 3D energy harvesting system and apply the Lagrange approach to derive the differential equations of motion. The electromechanical system consists of a mechanical resonator, piezoelectric transducer, and electrical circuit with load resistor. Here, a flexible and slender rod clamped at the bottom and loaded at the tip mass acts as the resonator. Moving in the 3D space, it enables the system to avoid the gravitational potential barrier of the straight vertical shape in case of buckling. This paper investigates the response of the rod deflection and the root-meansquare power output of selected vibration mode shapes of the rod with an attached tip mass.

Ghouli and Belhaq [15] investigate quasi-periodic vibration-based energy harvesting in a delayed van der Pol-Duffing oscillator coupled to a delayed piezoelectric mechanism. The authors work under the assumption that the time delay in the mechanical subsystem can be modulated around a mean value with a certain amplitude and frequency. The case of delay parametric 
resonance, for which the frequency of the modulation is nearly twice the natural frequency of the oscillator, is considered. The first- and second-step multiple scale methods are applied to obtain approximations of periodic and quasi-periodic solutions as well as the corresponding output powers. Bifurcation analysis is carried out to locate regions of existence of these solutions. The effects of various system parameters on the performance of quasi-periodic vibration-based energy harvesting are examined, and the advantage of using quasi-periodic vibrations to extract energy over a broad range of system parameters away from the resonance is illustrated. In closing, numerical simulations are run to validate the analytical predictions.

Ribeiro et al. [16] assess the nonlinear dynamic behavior of a system for energy harvesting using ocean wave motion. The system consists of a linear electromagnetic system coupled to a float excited by ocean waves. They analyze the average power as a function of parameters linked to the wave profile, the resistance load of the electromechanical motor, and the nonlinear parameters of the cubic spring. The nonlinear dynamic behavior is investigated with the aid of the maximum Lyapunov exponent, bifurcation diagrams, phase portraits, and Poincaré maps.

Felix et al. [17] implement a time signal distribution network with three nodes to establish a common time base, with adjustment for disturbances to the clock. The phase detection process generates a periodic oscillation called double-frequency jitter (DFJ). DFJ is inevitable in this context, appearing even with the PLL operating in the blocking range. Therefore, this work seeks to study the effect of the double-frequency oscillation on a network with signal transmission delay, its influence on the network's synchronous states, and the ability of oscillators to restore the clock's common time base when disturbed. Phase-locked loops, first proposed in the 1930s, are now taking on new relevance, given their role in time-base generation and detection either in integrated circuits or in smart grid power distribution systems. One important component of the PLL architecture is the phase detector. The phase detection function is a measure of phase/frequency errors in the phase-locked loop, with analog, hybrid, and digital implementations. In most of the classical literature, the phase detection function is implemented by a signal multiplier device that can be approximated by a sine function from the phase error. Additional simplifications made on the phase detection function are presented by Sanchez et al. [18], who provide a performance comparison between the multiplier, sine, and Duffing detectors.

Szmit [19] considers numerical and experimental methods in the free and forced vibrations of a rotating structure consisting of a rigid hub and three flexible beams. The finite element method is used to design the rotating structure and analyze its complex dynamic response. In the numerical calculations, both symmetric and de-tuned rotors are analyzed. Lastly, results obtained from ordinary differential equations and numerical simulations are compared with experimental tests.

MacLean et al. [20] numerically investigate the effects of actuator delay and actuation constraints on the performance of a modified integral resonant controller with integral tracking. Actuation delay and constraints naturally limit controller performance, so much so that they can cause instabilities. A 2-DOF drill string with a nonlinear bit-rock interaction model is analyzed. The control scheme is implemented on this system and analyzed under the effects of actuation delay and constraints, proving to be highly effective at coping with these limitations. The scheme is then compared to sliding-mode control and shown to be superior in many regimes of operation. Lastly, the scheme is analyzed in detail by varying its gains as well as several system parameters, most notably the actuation delay.

Tusset et al. [21] address the nonlinear dynamics and control of atomic force microscopy (AFM) considering fractional-order derivatives. Numerical simulations show the existence of chaotic behavior for some regions in the parameter space, the behavior of which is characterized by using the power spectral density and the 0-1 test. To bring the system from a chaotic state to a periodic one, the nonlinear saturation control (NLSC) and time-delayed feedback control (TDFC) techniques for the fractional-order systems are applied with and without accounting for the fractional-order derivative. Numerical results reveal the influence of the fractionalorder derivative on the dynamics of the AFM system. Consequently, certain phenomena arise, which are confirmed through detailed numerical investigations with the $0-1$ test. The NLSC and TDFC techniques are shown to be efficient in controlling the chaotic behavior of AFM in fractional-order derivatives.

In a companion paper, Ribeiro et al. [22] investigate a nonlinear dynamics model of AFM with the influence of a viscoelastic term. The mathematical model is based on non-resonant and almost linear responses, together with the deflection of the microcantilever, and considers the interaction forces between the atoms of the tip and the sample surface. The results reveal the influence of the viscoelastic term on the nonlinear dynamics of this model. The authors subsequently analyze the model using a fractional calculus with the RiemannLiouville operator derivative applied to the viscoelastic term, and thus assess the fractional nonlinear dynamics of the AFM system.

Tomé Manuel et al. [23] describe the mathematical modelling of the kinematics and dynamics of fourwheeled vehicles (light vehicles) to ensure that the vehicle design offers better drivability on local roads or highways. The mathematical modelling of the kinematics and dynamics (factors influencing vehicle performance) is proposed for traction on the front two wheels and for all four wheels, allowing us to classify the type of vehicle and where it can be implemented. Lastly, a computational simulation of kinematic variables that directly influence vehicle dynamics is presented and justified.

Hedrih [24] proposes and studies tools such as linear and nonlinear transformation of coordinates and 
angular velocity and intensity change in basic vectors of tangent space of a position vector of a material system kinetic point. In a curvilinear coordinate system, the coordinates of a geometrical or kinematical point are not the same as the coordinates of its corresponding position vector. Expressions of basic vectors of tangent space of kinetic point vector positions in generalized curvilinear coordinate systems are derived for orthogonal and nonorthogonal curvilinear coordinate systems alike. Expressions of change in these basic vectors over time are also derived. In turn, the geometrical (physical), covariant, and contravariant coordinates of the position vector of a kinetic mass point in a coordinate system determined by basic vectors of tangent space of this kinetic point vector position, and in generalized curvilinear coordinate systems, are determined. Original expressions of the angular velocity and velocity of dilatations for basic vectors of tangent space of kinetic point vector positions, both in generalized curvilinear coordinate systems and in a number of special orthogonal curvilinear coordinate systems, are derived.

Cveticanin [25] proposes a rotating vector method for the analysis of nonlinear oscillators using trigonometric functions. Working under the assumption that the nonlinearity is small, all terms of the series expansion of the function higher than the first are omitted. The rotating vectors for each mass are presented in the complex plane. In the paper, the suggested rotating vector procedure is applied to solve a three-degree-offreedom periodically excited metamaterial electromechanical system. The influence of the nonlinear stiffness of the flexible elastic beam, excited with a certain electric force, on the resonant properties of the system as a whole is investigated. It is shown that the influence of nonlinearity on the amplitude and phase of vibration is more significant for smaller values of the excitation frequency than for larger ones.

Hedrih [26] addresses the issues of phenomenological mapping and mathematical analogies of oscillatory regimes in hybrid discrete continuum systems of coupled deformable bodies. The systems consist of coupled deformable bodies like plates, beams, belts, or membranes connected by a viscoelastic nonlinear layer, modelled using continuously distributed elements of KelvinVoigt type with nonlinearity of the third order. Using the mathematical analogies, the similarities of structural models in systems of plates, beams, belts, or membranes become readily apparent. The structural models used consist of a set of two coupled nonhomogeneous partial nonlinear differential equations. The problems to solve are divided into space and time domains with the classical Bernoulli-Fourier method. In the time domain, the systems of coupled ordinary nonlinear differential equations are completely analog for different systems of deformable bodies and are solved by using the Krilov-Bogolyubov-Mitropolskiy asymptotic method. This paper presents a mathematical analytical calculus that can be applied to physically different systems, offering a powerful and useful tool for making the final conclusions between many input and output values. The conclusions regarding nonlinear phenomena in multibody system dynamics are revealed from the specific example of the double plate system's stationary and nonstationary oscillatory regimes.

Lastly, Khan et al. [27] examine the peristaltic transport of nano-fluid through a bio-fluidic channel under the influence of a magnetic field. The geometry of the channel is assumed to be inclined and Oldroyd fourconstant fluid to be flowing through it with Joule heating effects. The results obtained for stream function, velocity, wall shear stress, nanoparticle volume fraction, and temperature profile are demonstrated graphically to evaluate the physical aspects of emerging parameters such as the Hartman number, angle of inclination, phase angle, Brownian motion constant, nonuniform constant, and thermophoresis. Trapping phenomena are also prepared to help visualize the flow phenomena.

Acknowledgements The authors wish to express their appreciation for the support provided in the form of a grant from the National Council for Scientific and Technological Development (CNPq); project DIALOG 0019/DLG/2019/10 for 2019-2021 provided by the Ministry of Science and Higher Education of Poland; and CAPES [finance code 001, Grant Number 88882.306492/2018-01]. The authors and guest editors also wish to thank Sandrine Karpe for her invaluable assistance in editing this special issue.

\section{References}

1. R. Fellah, M.S. Azzaz, C. Tanougast, Design of a simple and low-cost chaotic signal generation circuit for UWB applications. Eur. Phys. J. Spec. Top. (2021). https:// doi.org/10.1140/epjs/s11734-021-00251-5

2. J.R.C. Piqueira, Melnikov's method applied to accidental phase modulation phenomenon. Eur. Phys. J. Spec. Top. (2021). https://doi.org/10.1140/epjs/ s11734-021-00229-3

3. H.H. Daum, A.M. Tusset, M.A. Ribeiro, G. Litak, A.M. Bueno, J.M. Balthazar, Analysis and chaos control of a four-dimensional magnetohydrodynamic model with hyperchaotic solutions. Eur. Phys. J. Spec. Top. (2021). https://doi.org/10.1140/epjs/s11734-021-00236-4

4. M.S. Azzaz, R. Fellah, C. Tanougast, R. Kaibou, Design and FPGA implementation of TRNG based on a new multi-wing attractor in Lorenz chaotic system. Eur. Phys. J. Spec. Top. (2021). https://doi.org/10.1140/ epjs/s11734-021-00234-6

5. S. Sun, D. Yan, M. Ji'e, X. Du, L. Wang, S. Duan, Memristor-based time-delay chaotic system with hidden extreme multi-stability and pseudo-random sequence generator. Eur. Phys. J. Spec. Top. (2021). https://doi. org/10.1140/epjs/s11734-021-00248-0

6. V. Piccirillo, Improving the dynamic integrity of a non-ideal oscillator via linear vibration absorber. Eur. Phys. J. Spec. Top. (2021). https://doi.org/10.1140/ epjs/s11734-021-00244-4

7. M.L.S. de Oliveira, P.B. Gonçalves, Predicting the dynamics of the human gait single support phase. Eur. Phys. J. Spec. Top. (2021). https://doi.org/10.1140/ epjs/s11734-021-00230-w 
8. J.C. Lacerda, J. Dias, C. Freita, E. Macau, Vulnerability and stability of power grids modeled by second order Kuramoto model - a mini review. Eur. Phys. J. Spec. Top. (2021). https://doi.org/10.1140/epjs/ s11734-021-00246-2

9. S.T.R. Rizvi, A.R. Seadawy, M.A. Ashraf, A. Bashir, M. Younis, D. Baleanu, Multi-wave, homoclinic breather, M-shaped rational and other solitary wave solutions for coupled-Higgs equation. Eur. Phys. J. Spec. Top. (2021). https://doi.org/10.1140/epjs/s11734-021-00270-2

10. C. Guo, A.C.J. Luo, Symmetric and asymmetric periodic motions of a nonlinear oscillator with a tuned mass damper inerter. Eur. Phys. J. Spec. Top. (2021). https://doi.org/10.1140/epjs/s11734-021-00235-5

11. A.K. Telem, K. Rajagopal, B. Ramakrishnan, On the dynamics of a system of two coupled van der pol oscillators subjected to a constant excitation force: effects of broken symmetry. Eur. Phys. J. Spec. Top. (2021). https://doi.org/10.1140/epjs/s11734-021-00232-8

12. M.E. Semenov, O. Reshetova, S.V. Borzunov, P.A. Meleshenko, Self-oscillations in a system with hysteresis: the small parameter approach. Eur. Phys. J. Spec. Top. (2021). https://doi.org/10.1140/epjs/ s11734-021-00237-3

13. A. Hedrih, K.R.S. Hedrih, Oscillations and stability of dynamics of hybrid biostructure. Eur. Phys. J. Spec. Top. (2021). https://doi.org/10.1140/epjs/ s11734-021-00240-8

14. M. Borowiec, M. Bochenski, G. Litak, A. Teter, Analytical model and energy harvesting analysis of a vibrating slender rod with added tip mass in three-dimensional space. Eur. Phys. J. Spec. Top. (2021). https://doi.org/ 10.1140/epjs/s11734-021-00241-7

15. Z. Ghouli, M. Belhaq, Energy harvesting in a delayinduced parametric van der Pol-Duffing oscillator. Eur. Phys. J. Spec. Top. (2021). https://doi.org/10.1140/ epjs/s11734-021-00243-5

16. M.A. Ribeiro, A.M. Tusset, W.B. Lenz, J.M. Balthazar, Dynamic Analysis of the non-linear behavior of an ocean buoy for energy harvesting. Eur. Phys. J. Spec. Top. (2021). https://doi.org/10.1140/epjs/ s11734-021-00273-z

17. R.O. Felix, A.M. Bueno, D.P.F. Correa, J.M. Balthazar, Analysis of double-frequency Jitter on time-delayed oscillators networks and perturbations adjustment in clock. Eur. Phys. J. Spec. Top. (2021). https://doi.org/ 10.1140/epjs/s11734-021-00239-1

18. R. Sanchez, J.R.C. Piqueira, A.M. Bueno, Comparing phase detectors in analog phase-locked loops. Eur. Phys. J. Spec. Top. (2021). https://doi.org/10.1140/ epjs/s11734-021-00245-3
19. Z. Szmit, Vibration, synchronization, and localization of three-bladed rotor - numerical studies and experimental studies. Eur. Phys. J. Spec. Top. (2021). https://doi. org/10.1140/epjs/s11734-021-00247-1

20. J. MacLean, V.S.S. Aphale, M. Wiercigroch, Feedback control method to suppress stick-slip in drillstrings featuring delay and actuation constraints. Eur. Phys. J. Spec. Top. (2021). https://doi.org/10.1140/ epjs/s11734-021-00228-4

21. A.M. Tusset, J.M. Balthazar, M.A. Ribeiro, W.B. Lenz, R.T. Rocha, Chaos control of an AFM Model in fractional order. Eur. Phys. J. Spec. Top. (2021). https:// doi.org/10.1140/epjs/s11734-021-00242-6

22. M.A. Ribeiro, A.M. Tusset, W.B. Lenz, I. Kirrou, J.M. Balthazar, Numerical analysis of fractional dynamical behavior of Atomic Force Microscopy. Eur. Phys. J. Spec. Top. (2021). https://doi.org/10.1140/epjs/ s11734-021-00271-1

23. C.J.T. Manuel, M.M.D. Santos, A.M. Tusset, Mathematical modeling attributed to kinematics and dynamics of a vehicle with 4 wheels. Eur. Phys. J. Spec. Top. (2021). https://doi.org/10.1140/epjs/ s11734-021-00238-2

24. K.R.S. Hedrih, Linear and non-linear transformation of coordinates and angular velocity and intensity change of basic vectors of tangent space of a position vector of a material system kinetic point. Eur. Phys. J. Spec. Top. (2021). https://doi.org/10.1140/epjs/ s11734-021-00226-6

25. L. Cveticanin, Rotating vector solving method applied for nonlinear oscillator. Eur. Phys. J. Spec. Top. (2021). https://doi.org/10.1140/epjs/s11734-021-00233-7

26. K.R.S. Hedrih, Structural analogies for hybrid discretecontinuum systems of deformable bodies coupled with non-linear layers. Eur. Phys. J. Spec. Top. (2021). https://doi.org/10.1140/epjs/s11734-021-00250-6

27. S.-S. Zhou, A. Abbasi, W. Farooq, M. Ijaz Khan, S. Ullah Khan, Peristaltic transport of nano-fluid through a bio-fluidic channel with Joule heating features: applications of physiological systems. Eur. Phys. J. Spec. Top. (2021). https://doi.org/10.1140/epjs/ s11734-021-00231-9 